\title{
Measurement of FEF25-75\% and FEF75\% does not contribute to clinical decision making
}

\author{
Philip H. Quanjer ${ }^{1}$, Daniel J. Weiner², Jeffrey J. Pretto ${ }^{3,4}$, Danny J. Brazzale ${ }^{5}$ and \\ Piotr W. Boros ${ }^{6}$
}

Affiliations: 'Depts of Pulmonary Diseases and Paediatrics-Pulmonary Diseases, Erasmus Medical Centre, Erasmus University, Rotterdam, The Netherlands. ${ }^{2}$ Children's Hospital of Pittsburgh, Pittsburgh, PA, USA. ${ }^{3}$ Dept of Respiratory and Sleep Medicine, John Hunter Hospital, Newcastle, ${ }^{4}$ School of Medicine and Public Health, University of Newcastle, Newcastle, and ${ }^{5}$ Dept of Respiratory and Sleep Medicine, Austin Hospital, Institute for Breathing and Sleep, Heidelberg, Australia. ' ${ }^{\circ}$ ung Function Laboratory, National Tuberculosis and Lung Diseases Research Institute, Warsaw, Poland.

Correspondence: P.H. Quanjer, Kervel 19, 7443 GT Nijverdal, The Netherlands. E-mail: pquanjerवziggo.nl

ABSTRACT The aim of this study was to determine the added value of measuring the forced expiratory flow at $25-75 \%$ of forced vital capacity (FVC) (FEF25-75\%) and flow when 75\% of FVC has been exhaled (FEF75\%) over and above the measurement of the forced expiratory volume in $1 \mathrm{~s}$ (FEV1), FVC and FEV1/ FVC ratio.

We used spirometric measurements of FEV1, FVC and FEF25-75\% from 11654 white males and 11113 white females, aged 3-94 years, routinely tested in the pulmonary function laboratories of four tertiary hospitals. FEF75\% was available in 8254 males and 7407 females. Predicted values and lower limits of normal, defined as the fifth percentile, were calculated for FEV1, FVC, FEV1/FVC ratio, FEF25-75\% and FEF75\% using prediction equations from the Global Lung Function Initiative.

There was very little discordance in classifying test results. FEF25-75\% and FEF75\% were below the normal range in only $2.75 \%$ and $1.29 \%$ of cases, respectively, whereas FEV1, FVC and FEV1/FVC ratio were within normal limits. Airways obstruction went undetected by FEF $25-75 \%$ in $2.9 \%$ of cases and by FEF $75 \%$ in $12.3 \%$ of cases.

Maximum mid-expiratory flow and flow towards the end of the forced expiratory manoeuvre do not contribute usefully to clinical decision making over and above information from FEV1, FVC and FEV1/FVC ratio.

@ERSpublications

Forced expiratory flow measurements do not contribute usefully to clinical decision making http://ow.ly/t6Xz1

For editorial comments see page 947.

Received: July 252013 | Accepted after revision: Sept 162013 | First published online: Sept 262013

Conflict of interest: None declared.

Copyright (CERS 2014 


\section{Introduction}

The forced expiratory vital capacity (FVC) manoeuvre is the fundamental manoeuvre of the test most frequently performed for assessing pulmonary function. Apart from the forced expiratory volume in $1 \mathrm{~s}$ (FEV1) and the FEV1/FVC ratio, a host of other indices have been derived from the FVC manoeuvre. These include peak expiratory flow, flows at 25\%,50\% and 75\% of the exhaled FVC and the forced expiratory flow at $25-75 \%$ of FVC (FEF25-75\%) (also called maximum mid-expiratory flow (MMEF)). The latter sets of flows, as well as the assessment of closing volume, were described as being more reproducible and more sensitive than FEV1 to the presence of "small airways disease" [1-3]. It was generally accepted that obstruction in small airways led to reduced flows at low lung volumes, leaving flows at high lung volumes much less affected, resulting in the characteristic concave flow-volume curve [4-6]. The prospect of detecting lung disease at an early stage has led to widespread measurement of $\mathrm{FEF} 25-75 \%$ and forced expiratory flow at 75\% of FVC (FEF75\%). However, the greater reproducibility of flows and better sensitivity of FEF25-75\% were also challenged [7, 8]. In addition, flows at a percentage of the FVC are sensitive to measurement errors in the FVC. Furthermore, as FVC and total lung capacity may be affected by disease, forced expiratory flows in patients will be measured at a different lung volume than in healthy subjects, rendering the use of predicted values derived from healthy subjects problematic. For the same reason serial measurements in a subject in whom the FVC has changed due to disease progression and/or therapeutic intervention should only be compared if they have been made at the same lung volume [9]. Finally, the hypothesis that reduced mid-expiratory flows were specific for small airways disease has been shown to be incorrect [10].

These limitations of instantaneous and mid-expiratory flows in clinical practice have led to recommendations to disregard any suspected abnormality in flows if the FEV1 and FEV1/FVC ratio are within normal limits [11-13]. However, it is suggested that in the presence of a borderline value of FEV1/ FVC, these tests may suggest the presence of airway obstruction [13]. Reporting of flow is also recommended for quality-control purposes in preschool children [14]. A search on PubMed using the keywords FEF25-75\%, MMEF and FEF75\% revealed that these indices were used in 1143 publications between 1975 and 2012, of which 385 were published since 2000 .

The purpose of this study is to investigate the extent to which the use of expiratory flows, namely the FEF25-75\% and FEF75\%, adds information to spirometry over and above that from FEV1, FVC and FEV1/FVC ratio.

\section{Materials and methods \\ Materials}

The study is based on 22767 consecutive patients routinely tested in the pulmonary function laboratories of the Children's Hospital of Pittsburgh (Pittsburgh, PA, USA), the Austin Hospital (Heidelberg, Australia) (Australia 1), the John Hunter Hospital (Newcastle, Australia) (Australia 2) and the National Research Institute of Tuberculosis and Lung Diseases (Warsaw, Poland). The studies were carried out between January 2010 and December 2011 (USA), August 2008 and June 2012 (Australia 1), January 2001 and May 2012 (Australia 2) and April 2009 and June 2012 (Poland). Spirometry was performed by trained respiratory therapists and scientists in accordance with internationally agreed standards applied at the time of data collection $[15,16]$, and only baseline or pre-bronchodilator data was included in the analysis.

The data comprised consecutively collected test results from patients referred for lung function assessment for clinical purposes. The bulk of the subjects in the USA dataset were referred for asthma control and suspected asthma, followed by cystic fibrosis, cough, dyspnoea or miscellaneous conditions, in that order. The study is limited to people of European ancestry because other ethnic groups were poorly represented.

TABLE 1 Numbers of males and females and age ranges in the four datasets

Male

\begin{tabular}{lccccc}
\cline { 2 - 3 } \cline { 5 - 6 } & Subjects & Age years & & Subjects & Age years \\
\hline Australia 1 & 1418 & & & & \\
Australia 2 & 1980 & $8.3-93.6$ & & 1401 & $6.5-94.6$ \\
Poland & 5662 & $18.0-91.0$ & & 2304 & $9.1-94.6$ \\
USA & 2594 & $3.8-65.0$ & & 2225 & $18.0-92.0$ \\
\hline
\end{tabular}

Data are presented as $\mathrm{n}$ or range.

Female 
TABLE 2 Z-scores for males and females for spirometric variables in the four datasets

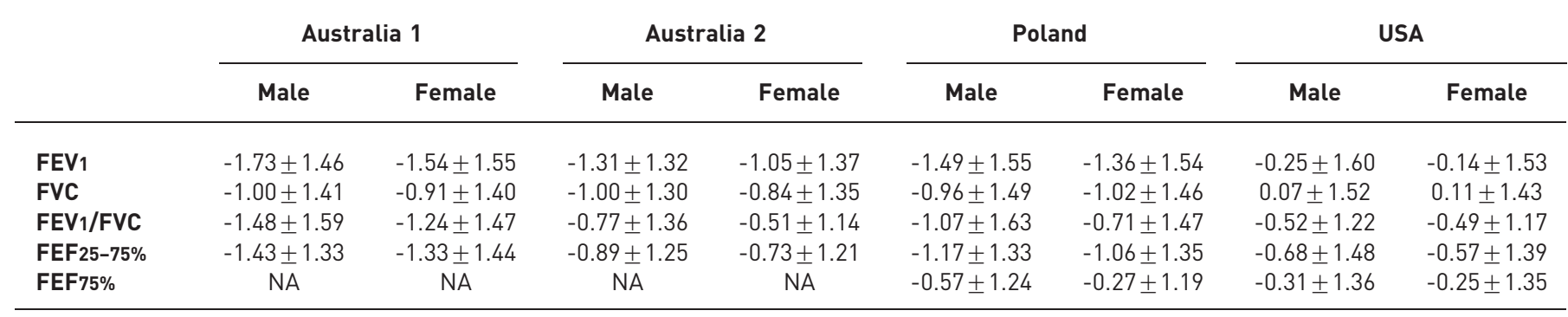

Data are presented as mean \pm SD. FEV1: forced expiratory volume in $1 \mathrm{~s}$; FVC: forced vital capacity; FEF25-75\%: forced expiratory flow at $25-75 \%$ of FVC; FEF75\%: forced expiratory flow at $75 \%$ of FVC; NA: not available.

The de-identified datasets were comprised of data on age, height, sex, ethnic group, FEV1, FVC, FEV1/FVC and FEF25-75\% values. Data on FEF75\% were only available in datasets from Poland and the USA. FEF25-75\% and FEF75\% were taken from the FVC manoeuvre with the highest sum of FEV1 and FVC [15]. If serial measurements had been performed on the same individual, only the first test result was included for analysis.

This study is a retrospective analysis of de-identified data, obviating the need for approval from local ethics committees. Nonetheless, separate approval was obtained from the institutional review board of the Children's Hospital of Pittsburgh (PRO12100285) and from the local ethics committees of the Australian laboratories.

\section{Methods}

Predicted values and z-scores for the various indices were derived using prediction equations from the Global Lung Function Initiative (GLI-2012) [17] using specialised software [18]. Plots were made of the Z-scores for FEV1/FVC ratio, FEF75\% and FEF25-75\%.

In males and females a best fit was obtained for height as a function of age using statistical modelling software (GAMLSS version 4.2-4; Generalized Additive Models for Location, Scale and Shape, www.gamlss. org). Heights outside \pm 3 z-scores from the mean were regarded as outliers. Z-scores for FEV1/FVC ratio, FEF25-75\% and FEF75\% $<-1.645$ were considered abnormal. Data analysis was performed using the statistical software R (version 3.0.1; The R Project for Statistical Computing, www.r-project.org).

\section{Results}

The numbers of males and females and age ranges in the four datasets are shown in table 1. 68 males and 67 females $(0.59 \%$ of the total study population) were very short or very tall for age, leading to extreme $\mathrm{z}$ scores for spirometric indices; these data were considered outliers and excluded, leaving 22632 spirometry results for analysis. The overall prevalence of airways obstruction, defined as FEV1/FVC ratio less than the lower limit of normal (LLN), was 26.4\%; on the basis of FEV1 \% predicted, using the American Thoracic Society (ATS)/European Respiratory Society (ERS) grading system [13], 8.85\% were classified as mild, $4.34 \%$ as moderate, $4.19 \%$ as moderately severe, $5.45 \%$ as severe and $3.56 \%$ as very severe airways obstruction. A "spirometric restrictive pattern" (where FEV1/FVC $>$ LLN and FVC $<$ LLN) was found in $15.3 \%$ of the total group.

The mean z-scores for males and females for the various spirometric variables in the four datasets are shown in table 2. The LLN for FEF25-75\% and FEF75\% varied between 35-67\% and 31-56\%, respectively, declining steeply with age. The relationship between the z-scores for the FEV1/FVC ratio and FEF25-75\% is shown in figure 1a; no differences in this relationship could be discerned between males and females. Of the 22767 subjects, there were $1862(8.18 \%)$ that showed a FEF25-75\% below the LLN but a FEV1/FVC ratio in the normal range (quadrant Q3 in fig. 1a). In 1235 (66\%) of these individuals this was associated with a FVC below the LLN (figs 1c and 2). 2.87\% of test results revealed a FEF25-75\% in the normal range, but FEV1/ FVC $<$ LLN (suggesting airways obstruction) as indicated in quadrant Q1 in figure 1a. The percentage of subjects in whom FEF25-75\% was less than LLN but FEV1, FVC and FEV1/FVC were in the normal range is displayed as a function of age in figure 2. After adjusting for the $\mathrm{z}$-score for the FEV1/FVC ratio, the z-score for FEF $25-75 \%$ was significantly lower $(-0.12)$ in the Polish than in the other datasets. 

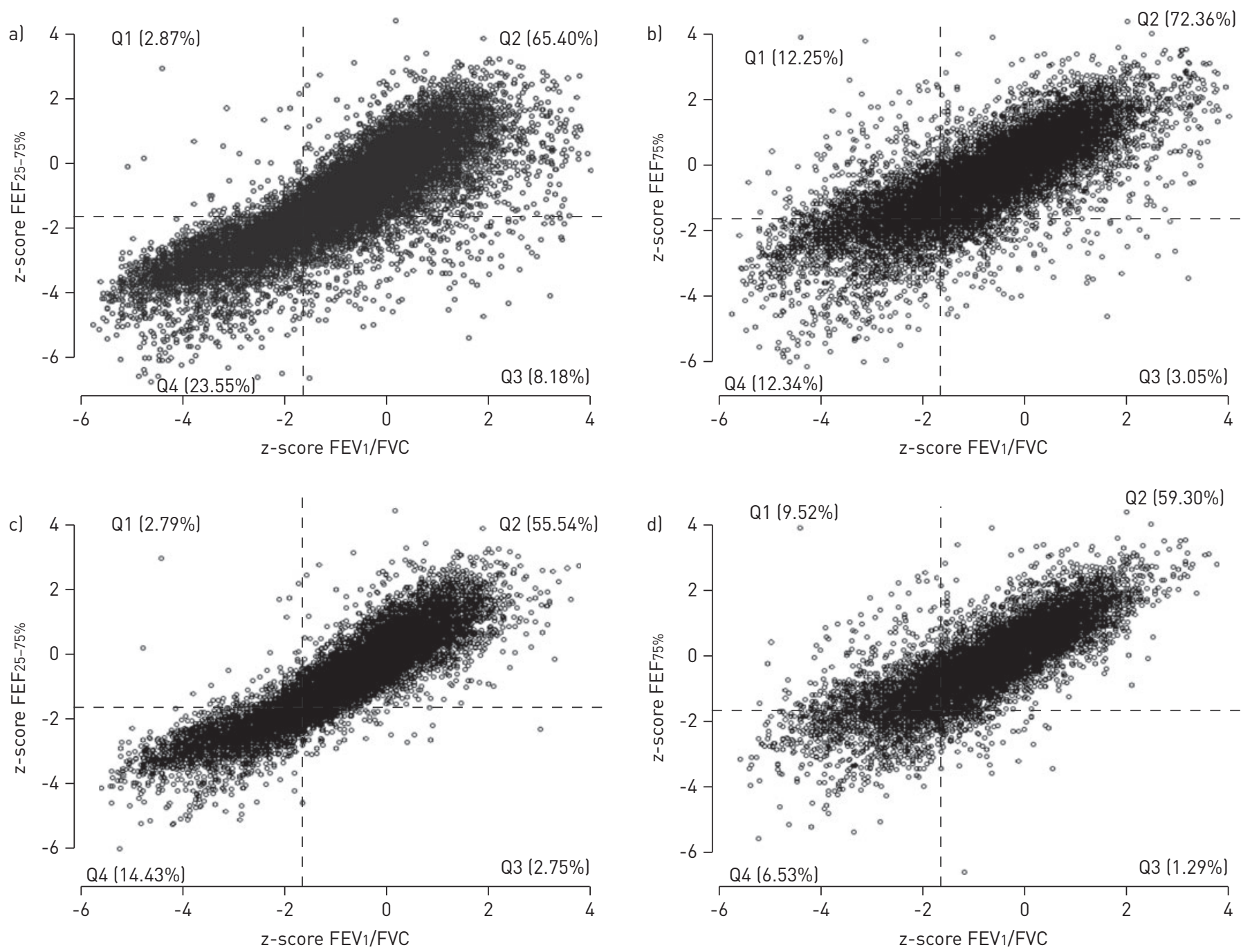

FIGURE 1 Relationship between the z-scores of a) forced expiratory flow at 25-75\% of forced vital capacity (FVC) (FEF25-75\%) and b) forced expiratory flow at $75 \%$ of FVC (FEF75\%) with that of the forced expiratory volume in $1 \mathrm{~s}(\mathrm{FEV} 1) / \mathrm{FVC}$ ratio. Data in figures c) and d) are subsets of a) and b), respectively, where the FVC is above the lower limit of normal. The dashed lines indicate the lower limits of normal $(\mathrm{z}=-1.645)$. The percentage values indicate the percentage of the total 22767 test results for FEF25-75\% and 15661 test results for FEF75\% that fall into each quadrant (Q1-Q4).

Data on FEF75\%were available in 15661 subjects and there was a similar relationship with FEV1/FVC ratio as with FEF25-75\% (fig. 1b). There were 477 cases $(3.05 \%$ of the total) where FEF75\% was reduced $(<\mathrm{LLN})$ but there was a normal FEV1/FVC ratio (Q3 in fig. 1b), and the majority of these cases (58\%) showed a reduced FVC (fig. 1d). Compared with $\mathrm{FEF}_{25-75 \%}$ there were significantly more results where FEV1/FVC ratio was reduced but FEF75\% was not (12.25\% of the total). After adjusting for the $\mathrm{z}$-score for FEV1/FVC ratio, the z-score for FEF75\% was significantly lower $(-0.17)$ in the USA than in the Polish dataset.

Thus, only $2.75 \%$ of the total number of test results showed a reduced FEF25-75\% with both FEV1/FVC and FVC within the normal range. The corresponding value for FEF75\% was lower, at only $1.29 \%$ of the total. Of these cases, about half showed a FEV1/FVC ratio close to the LLN (within 0.25 z-values). When we reviewed the discordances in a random sample of 100 cases, $67 \%$ of them were found to have an artefact or submaximal exhalation which could affect results $(71 \%$ in the $3-10$ years age range and $29 \%$ in the 10-20 years age range).

A normal FEF25-75\% associated with an abnormal FEV1/FVC ratio was rare (2.87\%, quadrant 1 in fig. 1a). These cases were characterised by a low FEV1 (mean z-score -0.79), an above-average FVC (mean z-score 0.55 ) and mostly mild airways obstruction (87.2\% using the ATS/ERS grading system [13]). In 12.25\% of cases a normal FEF75\% was associated with an abnormally low FEV1/FVC ratio; characteristically there was more severe airways obstruction (mean z-score for FEV1 - 1.87, for FVC -0.52 and $49.7 \%$ cases of mild airways obstruction). 
FIGURE 2 Percentage of subjects, by 2year age groups, in whom the forced expiratory flow at $25-75 \%$ of forced vital capacity (FVC) (FEF25-75\%) was below the lower limit of normal, whereas forced expiratory volume in $1 \mathrm{~s}$ (FEV1), FVC and FEV1/FVC ratio were within the normal range.

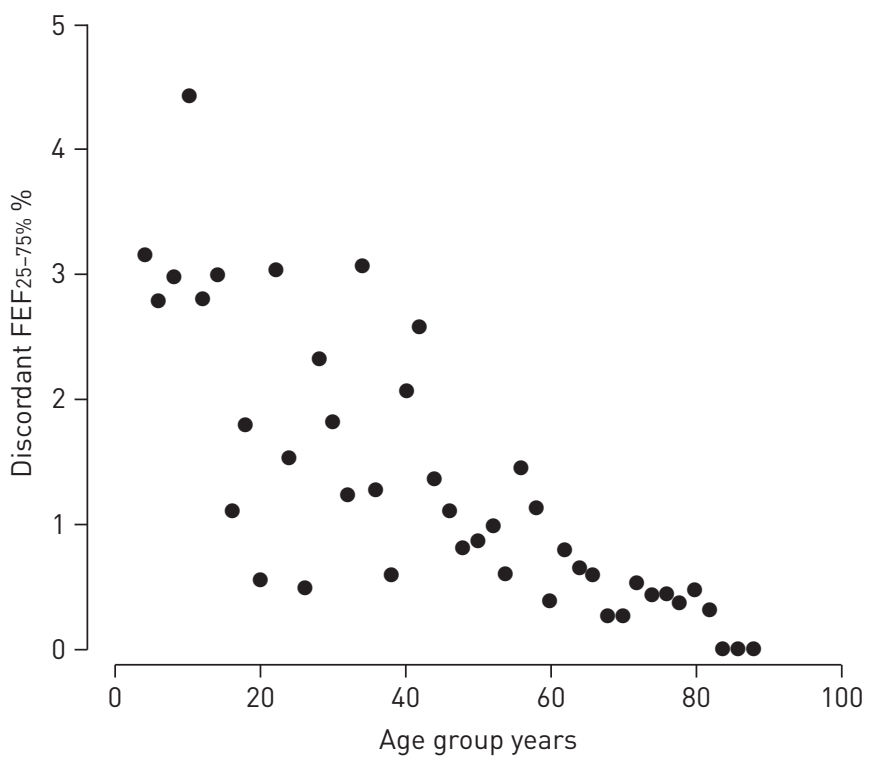

As the flow-volume curve clearly demonstrates, expiratory flows are highly dependent upon the lung volume at which they are determined. Similar to FEV1 values, they were also expressed as a fraction of the FVC to take this dependency into consideration. The relationship between FEF25-75\%/FVC ratio and FEF75\%/FVC ratio with FEV1/FVC ratio was curvilinear; however, log-transforming the data linearised the relationship and stabilised the variance, as shown in figure 3.

Forced expiratory flows are commonly presented as a percentage of the mean predicted value. Figure 4 depicts the LLN (5th percentile, at z-score of -1.645) for FEF25-75\% and FEF75\% as per cent predicted, using the GLI-2012 equations and median height for age in males and females.

\section{Discussion}

To our knowledge this is the first study that has systematically investigated whether the use of maximum mid-expiratory flow and instantaneous flows provides additional information beyond that provided by traditional spirometric indices (FEV1, FVC and FEV1/FVC ratio) in detecting lung function impairment. Our findings in a large clinical dataset show that airways obstruction, defined by an abnormally low FEV1/ FVC ratio, goes undetected by the $\mathrm{FEF} 25-75 \%$ and $\mathrm{FEF} 75 \%$ in $2.9 \%$ and $12.3 \%$ of cases, respectively. We have found that $\mathrm{FEF} 25-75 \%$ is reduced in the presence of a normal FEV1/FVC ratio in $8.2 \%$ of cases. Only $3.0 \%$ of cases revealed an abnormally low FEF75\% with a normal FEV1/FVC ratio. However, a large majority of these discrepant findings occurred when the FVC was abnormally low. As such, in only a very small minority of cases was a low $\mathrm{FEF} 25-75 \%$ or $\mathrm{FEF} 75 \%$ associated with normal FEV1/FVC ratio and a normal FVC (2.8\% and $1.3 \%$, respectively). Of these discordant cases, we identified possible artefact or unsatisfactory FVC efforts in $\sim 63 \%$ of cases.

It is commonly perceived that the $\mathrm{FEF} 25-75 \%$ is diagnostically more helpful in a paediatric population than in adults. Indeed, we did find a trend of a higher prevalence rate of an abnormally low FEF25-75\% when $\mathrm{FEV} 1, \mathrm{FVC}$, and FEV1/FVC ratio are in the normal range in youngsters than in adults (fig. 2), but even then the rate is very low at only $3 \%$. It is difficult to always obtain high quality measurements in children. Indeed, in a random sample of 100 children with discordant findings in the 3-10-year and 10-20-year age ranges, $71 \%$ and $29 \%$, respectively, were found to be associated with flow-volume curves with an artefact or submaximal exhalation or inhalation, which could affect results. The very low incidence of discordant findings argues against the notion that forced expiratory flows are more sensitive to small airways obstruction than other spirometric indices. We suggest that these infrequent occurrences where flow indices result in discordant findings from conventional spirometric indices probably represent statistical noise and biological variability associated with spirometry, and in particular with the high reliance of expiratory flow indices on the valid measurement of FVC. We would also suggest that such findings should prompt a critical review of the quality of the FVC manoeuvre. The practical implication is that the flow indices contribute little or no additional information over and above that provided by the FEV1 and FVC and, therefore, do not add to the diagnostic arsenal in detecting airways obstruction. 

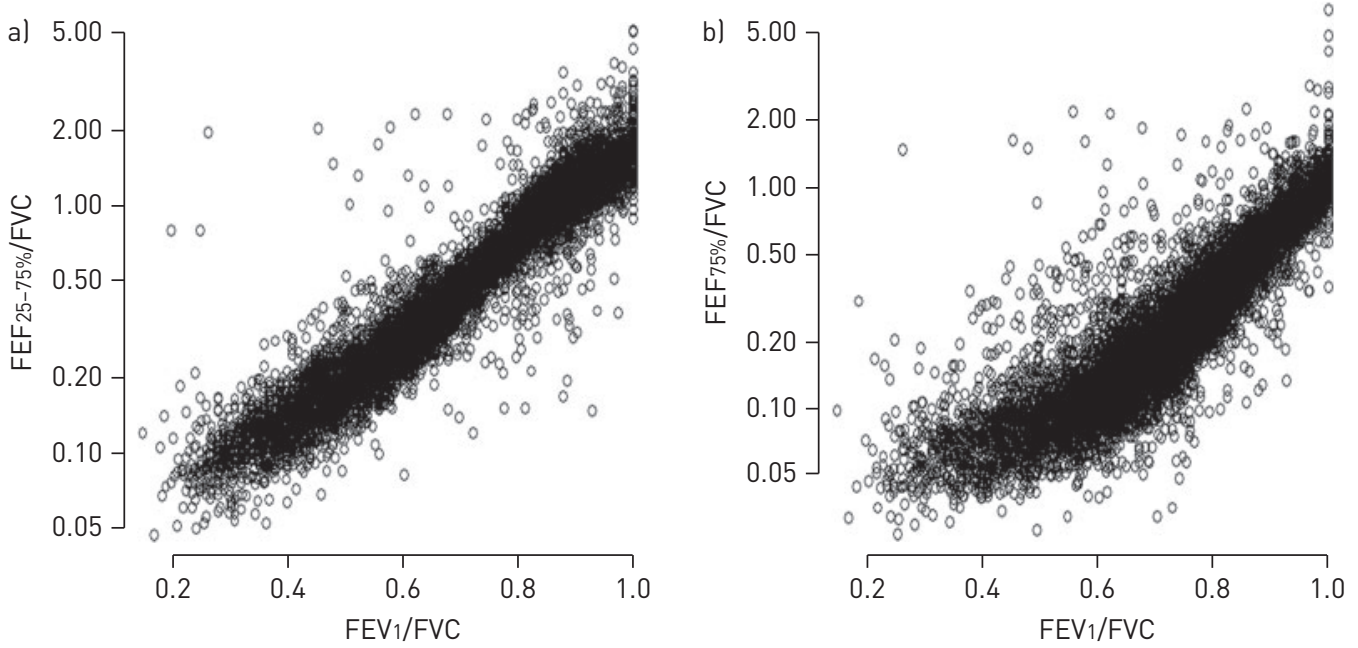

FIGURE 3 Relationship between a) forced expiratory flow at 25-75\% of forced vital capacity (FVC) (FEF25-75\%) and b) forced expiratory flow at 75\% of FVC (FEF75\%) and forced expiratory volume in $1 \mathrm{~s}$ (FEV1), both standardised for the FVC. Note that the $y$-axis is log-transformed.

The frequent use of these flow indices is based on the notion that airways obstruction affects flows towards the end of a forced expiratory manoeuvre much more than at the start of the FVC manoeuvre, because at low lung volumes lung elastic recoil is low and airway dimensions are small. Hence, such flows are thought to be more sensitive to small airways obstruction than the FEV1. This misconception arose at least in part because MCFADDEN and LINDEN [1] based their conclusions on the erroneous, yet still popular, idea that $80 \%$ pred denotes the LLN for any marker of lung function. This ignores the considerable age related difference in variability of lung function with age [17] and has led to considerable overdiagnosis of abnormality. The LLN for FEF $25-75 \%$ is $67 \%$ of predicted mean in childhood and $35 \%$ in those aged $\geqslant 80$ years; corresponding values for $\mathrm{FEF} 75 \%$ are $56 \%$ and $31 \%$. Indeed, as early as 1988, FLENLEY [10] contested the view that mid-expiratory flows were specific for small airways disease. By inference, partitioning into large and small airways obstruction in chronic lung diseases on the basis of spirometric test results is not warranted.

The dependency of forced expiratory flow on lung volume is immediately clear from flow--volume curves, unlike the mechanisms responsible for determining such flows. During a forced expiration the high pleural pressure leads to dynamic compression of intrathoracic airways. Flow through compressed airways is then determined by wave speed limitation, i.e. when local gas velocity is equal to the speed of propagation of pressure waves in the airways; the location of the flow-limiting segment (choke point) is determined by the interplay between lung elastic recoil and airway compliance [19, 20]. As more volume is expelled, lung elastic recoil and pleural pressure diminish, which allows the choke point to settle upstream in airway

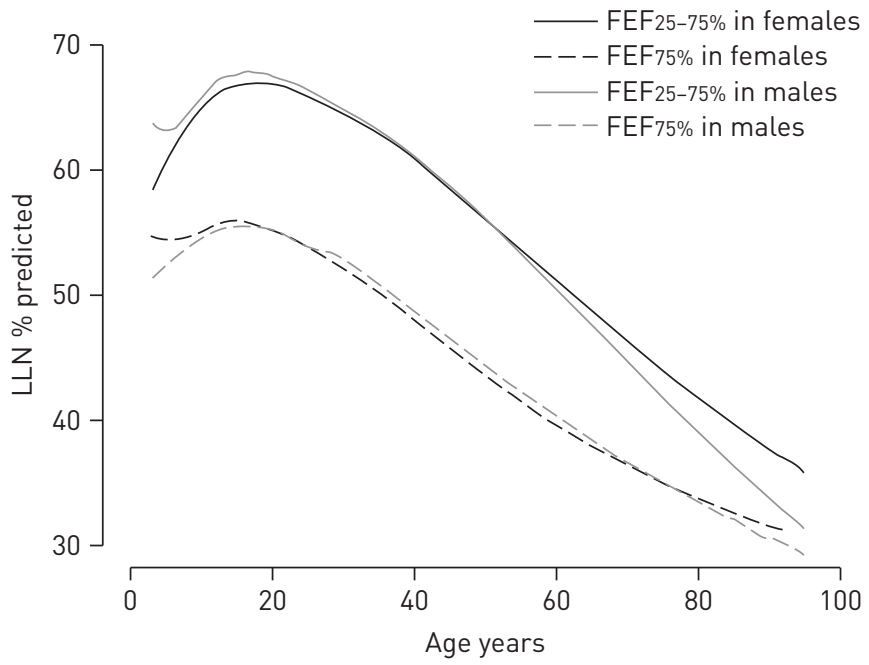

FIGURE 4 The lower limit of normal (LLN) as a percentage of the predicted value for forced expiratory flow at 25$75 \%$ of forced vital capacity (FEF25-75\%) and forced expiratory flow at $75 \%$ of forced vital capacity (FEF75\%) in females and males. 
segments with pressure-area characteristics that allow lower flow [21, 22]. It follows that forced expiratory flows are highly dependent on lung volume so that, like the FEV1 (the average flow over the first second of the forced expiration), they should be standardised for true lung volume, which is the total lung capacity. This is usually not feasible, and hence the vital capacity is used as a proxy for lung size. The FEV1/FVC ratio is dimensionally s ${ }^{-1}$ and represents the lung emptying rate. As our analysis has shown, standardising the FEF25$75 \%$ and $\mathrm{FEF} 75 \%$ in a similar fashion by expressing them as a ratio of the FVC, reveals high correlations with the FEV1/FVC ratio (fig. 3), signifying that the information content of these indices is very similar.

Obstructive respiratory disease with increasing residual volume causes flow at a fixed percentage of the FVC to be measured closer to the total lung capacity (which may be increased due to disease) than in a reference population; this partly masks a fall in forced expiratory flows due to airways obstruction and will diminish the sensitivity in diagnosing obstructive lung disease. In addition, normal reference ranges for forced expiratory flows reflect between-subject variability both in flows and in the FVC. This leads to much higher coefficients of variation than for FEV1, FVC or their ratio and, therefore, to larger reference intervals around predicted values (fig. 4). Variability might have been somewhat smaller if flows had been taken from the envelope of flow-volume curves [12,23], but in this study it was taken from the FVC manoeuvre with the highest sum of FEV1 and FVC in accordance with recommended guidelines [15]. Such wide intervals render these indices essentially worthless for diagnostic purposes [12, 17, 24]. This also highlights that using $80 \%$ pred as the LLN for lung function indices lacks any scientific basis and leads to highly biased and erroneous clinical decisions. The use of a lower limit defined as the lower 5th or other percentile of the distribution in a healthy population of nonsmokers is, therefore, the recommended procedure [12, 14, 17, 25-28]. Z-scores indicate the number of age-specific standard deviations that a measured value differs from the predicted value and, unlike using a fixed percentage of predicted as the basis for interpretation of lung function, they are free of bias due to age, height, sex and ethnic group [12, 14, 17, 25-28] and were, therefore, used in this study.

This study is based on a large number of data from patients referred to tertiary hospitals for suspected or known lung disease. The relationship between the z-score for FEV1 and that for flows differed only slightly, albeit significantly, between centres, indicating that our findings are robust and applicable to children and adults with a wide range of normal and abnormal lung function test results. However, the very large number of data precluded rigorous post hoc quality control of all original spirograms. In routinely collected data there will be a proportion where data are clinically useful and therefore accepted, but where the FVC manoeuvres do not meet stringent quality criteria. As shown by this study, a low FEF $25-75 \%$ or FEF75\% when the FVC, FEV1 and FEV1/FVC ratio are within normal limits is rare and should lead to reviewing whether the FVC manoeuvre was performed correctly.

\section{Conclusions}

Measurements of $\mathrm{FEF} 25-75 \%$ and $\mathrm{FEF} 75 \%$ are highly correlated with conventional spirometric indices, leading to minimal discordance in classifying test results. Most reductions in FEF25-75\% and FEF75\% measurements in the absence of classically defined airways obstruction using FEV1/FVC data result from reduced lung volume rather than from airways disease. The low incidence of abnormal expiratory flows with normal FEV1 and FVC values may reflect measurement "noise". These data suggest that maximum midexpiratory flow and flow towards the end of the forced expiratory manoeuvre do not contribute usefully to clinical decision making.

\section{References}

1 McFadden ER Jr, Linden DA. A reduction in maximum mid-expiratory flow rate. A spirographic manifestation of small-airways disease. Am J Med 1972; 52: 725-737.

Gelb AF, Zamel N. Simplified diagnosis of small-airway obstruction. N Engl J Med 1973; 288: 395-398. McCarthy DS, Spencer R, Greene R, et al. Measurement of "closing volume" as a simple and sensitive test for early detection of small airway disease. Am J Med 1972; 52: 747-753.

Bates DV. Respiratory Function in Disease. 3rd Edn. Philadelphia, WB Saunders, 1989.

Wilson AF, ed. Pulmonary Function Testing: Indications and Interpretations. Orlando, Grune \& Stratton, 1985. Pride NB. Macklem PT. Lung mechanics in disease. In: Macklem PT, Mead J, eds. Handbook of Physiology. The Respiratory System. Mechanics of Breathing. Section 3, Vol. III, part 2. Bethesda, American Physiological Society, 1986; pp 659-692.

Gelb AF, William AJ, Zamel N. Spirometry. FEV1 vs FEF25-75 percent. Chest 1983; 84: 473-474.

Sobol BJ, Emirgil C. Subject effort and the expiratory flow rate. Am Rev Respir Dis 1964; 89: 402-408.

Sherter CB, Connolly JJ, Schilder DP. The significance of volume-adjusting the maximal midexpiratory flow in assessing the response to a bronchodilator drug. Chest 1978; 73: 568-571.

10 Flenley DC. Chronic obstructive pulmonary disease. Dis Mon 1988; 34: 537-599.

11 Lung function testing: selection of reference values and interpretative strategies. Am Rev Respir Dis 1991; 144: 1202-1218.

12 Quanjer PH, Tammeling GJ, Cotes JE, et al. Lung volumes and forced ventilatory flows. Report Working Party Standardization of Lung Function Tests, European Community for Steel and Coal. Official Statement of the European Respiratory Society. Eur Respir J 1993; 6: Suppl. 16, 5-40.

13 Pellegrino R, Viegi G, Brusasco V, et al. Interpretative strategies for lung function tests. Eur Respir J 2005; 26: 948-968. 
14 Beydon N, Davis SD, Lombardi E, et al. An official American Thoracic Society/European Respiratory Society statement: pulmonary function testing in preschool children. Am J Respir Crit Care Med 2007; 175: 1304-1345.

15 Miller MR, Hankinson J, Brusasco V, et al. Standardisation of spirometry. Eur Respir J 2005; 26: 319-338.

16 Standardization of spirometry, 1994 update. American Thoracic Society. Am J Respir Crit Care Med 1995; 152: $1107-1136$.

17 Quanjer PH, Stanojevic S, Cole TJ, et al. Multi-ethnic reference values for spirometry for the 3-95-yr age range: the global lung function 2012 equations. Eur Respir J 2012; 40: 1324-1343.

18 Quanjer PH, Stanojevic S, Cole TJ, et al. GLI-2012 data conversion software. www.lungfunction.org/tools.html Date last updated: October 12, 2013.

19 Pedersen OF, Nielsen TM. The critical transmural pressure of the airway. Acta Physiol Scand 1976; 97: 426-446.

20 Dawson SV, Elliott EA. Wave-speed limitation on expiratory flow - a unifying concept. J Appl Physiol Respir Environ Exerc Physiol 1977; 43: 498-515.

21 Jones JG, Fraser RB, Nadel JA. Prediction of maximum expiratory flow rate from area-transmural pressure curve of compressed airway. J Appl Physiol 1975; 38: 1002-1011.

22 Dawson SV, Elliott EA. Use of the choke point in the prediction of flow limitation in elastic tubes. Fed Proc 1980; 39: 2765-2770.

23 Schrader PC, Quanjer PH, van Zomeren BC, et al. Selection of variables from maximum expiratory flow-volume curves. Bull Eur Physiopathol Respir 1983; 19: 43-49.

24 Hansen JE, Sun XG, Wasserman K. Discriminating measures and normal values for expiratory obstruction. Chest 2006; 129: 369-377.

25 Sobol BJ, Sobol PG. Per cent of predicted as the limit of normal in pulmonary function testing: a statistically valid approach. Thorax 1979; 34: 1-3.

26 Miller MR, Pincock AC. Predicted values: how should we use them? Thorax 1988; 43: 265-267.

27 Stanojevic S, Wade A, Stocks J, et al. Reference ranges for spirometry across all ages: a new approach. Am J Respir Crit Care Med 2008; 177: 253-260.

28 Miller MR, Quanjer PH, Swanney MP, et al. Interpreting lung function data using $80 \%$ predicted and fixed thresholds misclassifies more than $20 \%$ of patients. Chest $2011 ; 139 ; 52-59$. 